\title{
Types of Evidence Cited in Australian Government Publications
}

\author{
Samantha Vilkins ${ }^{1}$ and Will J Grant ${ }^{2}$
}

\author{
${ }^{1}$ Australian National Centre for the Public Awareness of Science, Australian National University, \\ orcid.org/ 0000-0002-7479-2683 \\ ${ }^{2}$ Australian National Centre for the Public Awareness of Science, Australian National University. \\ Corresponding Author Will Grant, will.grant@anu.edu.au , +61450105489, orcid.org/0000-0001-9674- \\ 6488
}

\begin{abstract}
Demand on researchers to justify the impact of their work outside academia is increasing. Both increasing research use in policy and measuring current use are multi-faceted problems, though there are many potential benefits to researchers and policymakers alike. This bibliometric study aimed to gain insight into the research and reference practices of Australian policymakers, and investigate how this approach compares to previous interview and survey studies. We analysed 4649 references from 80 government publications from eight departments from 2010 to 2017, including references to 1836 articles from peer-reviewed journals, noting each author, title, year, parent publication, source type and access level. The number and type of evidence sourced varied per publication, with the most common sources being peer-reviewed journal articles, federal government reports, and Australian business information. This differs from previous large-scale qualitative studies which found policymakers are most likely to speak directly to colleagues for information, and far less inclined to seek out academic research. The study also found a possible increased chance for academic research to be cited if it was open access. Despite criticisms of citation analysis, at least in the field of research utilisation we cannot solely rely on interview or survey data, as cited evidence use differs from reported evidence use. Both the characteristics of evidence sources in policy and the effect of open access publishing on research use in policy are clearly worth investigating further, particularly longitudinally, which would require increased accessibility of government publications.
\end{abstract}

\section{Introduction}

\section{Research impact in policy}

Researchers are increasingly required to demonstrate the impact of their work (Dolenc, Hünenberger and Renn, 2016). This is a notoriously difficult problem even within their own research communities, as impact is complex and at times intangible (King's College London and Digital Science, 2015; Wallin, 2005). The influence of research in spheres outside the research sector, such as in the world of policy making, is even more difficult to measure (Universities Australia, 2016). Yet capturing this impact on policy has significant potential benefits, including showing the impact of research on real- 
world settings, and building a better case for support for researchers and institutions or even broader research directions (Department of Industry Innovation and Science, 2017). Beyond this, there are also benefits to policymakers, who have reasoned that research helps to depoliticise new policies, cutting through political indecision or public uncertainty (Department of Education Training \& Youth Affairs, 2000). However, with the pace and voracity of modern scientific endeavour, a lack of relevant information for policymakers is no longer the concern it may have once been (Rich and Oh, 2000; Head et al., 2014). Part of increasing research impact in policy is understanding these competing sources of available evidence and the part they play in the policymaking process (Ouimet and Ziam, 2009).

For the past fifty years, the de facto measure for impact has been citation counts, or extensions thereof (Bollen et al., 2005). The concept of citations as a surrogate for impact and quality is deeply embedded into academic culture, with the majority of studies of academic research impact, as well as advice to researchers, speaking in the language of being "highly-cited" (Norris, Oppenheim and Rowland, 2008; Levitt and Thelwall, 2008; Deakin University, 2016; Curtin University, 2017). These metrics rely on databases such as the Scientific Citation Index, which does not include publications outside of peer-reviewed journals (Clarivate Analytics, 2017). Saha (2003) explains that if indexed citations are "votes" by researchers for journals or articles, then the votes of policymakers and other users of scientific research who do not publish peer-reviewed articles would not be easily counted. Consequently, previous studies of research impact specifically in policy have relied on extensive interview and survey data. These studies reveal how policymakers view their own processes and research used for policy, but without analysing citations, we ignore the existing, visible acknowledgements of research use and a crucial public stage of the policymaking process. This study aims to map the current uses of evidence by policymakers in Australia by analysing the citations of recent Australian Government publications.

\section{Studies of research use by policymakers}

Some broad trends regarding attitudes and practice can be seen from previous studies of research use in policymaking both in Australia and internationally. Two studies, one consisting of interviews with 60 federal and 419 state and local policymakers working in mental health policy in the United States (Rich and Oh, 2000), and the other a survey of 2084 public servants from various agencies in Australia (Head et al., 2014), found a major factor in research use seems to be organisational contexts.

Policymakers must make decisions that work in the interest of their organisation while being strongly influenced by organisational culture and perspectives towards research use. Responses such as "academic research results are considered relevant by my colleagues", "research is important in my professional field", and "policy making is based on sound evidence" were significant predictors of research use (Head et al., 2014). Lester's (1993) survey of 113 policymakers from hazardous waste management, economic development, welfare and education state agencies in the United States found some variance according to individual researcher characteristics, such as education level and experience, but Head et al. (2014) concluded that these organisational contexts were a stronger factor, at least in their Australian population. Similarly, organisational influence may be linked to the clear differences in research use across policy domains found by Amara, Ouimet and Landry (2004) in their survey of 833 Canadian government officials, and why there is no strong difference seen between similar departments in state or federal comparisons (Head et al., 2014). The policy domains of language, culture and immigration, justice and native affairs had the lowest use of academic research, while the domains of education and information technology had the highest (Amara, Ouimet and Landry, 2004). 
Different types of research are used at different stages of the policymaking process (Weiss, 1980; Lester, 1993). While Weiss developed this primarily from a series of interviews of 155 government officials working in mental health policy in the United States, Lester's (1993) survey came to the same conclusion, meaning it is likely true across policy domains. The different process stages can be broadly categorised in terms of three descriptions for research use: 'instrumental' use is direct and measurable use for policy; 'conceptual' use in indirect but rather affects thinking over a longer period of time; 'symbolic' use is when specific findings are selected for rhetorical or political argument (Weiss, 1980; Amara, Ouimet and Landry, 2004). According to Weiss (1980), instrumental use is rare and difficult to measure, while symbolic use is the most common. Amara, Ouimet and Landry's (2004) survey found $40.4 \%$ of government respondents described university research as at least moderately important for instrumental purposes, $59.5 \%$ as at least moderately important for conceptual purposes, and $51.8 \%$ for symbolic purposes.

In Australia, there have been specific studies on policymakers' preferred research methods and sources. Federal respondents to Head et al's (2014) survey ranked the "most important" (94\%) source as the knowledge of their immediate colleagues, with university researchers being the first external source listed at 70\%. The most important method for obtaining evidence was "searching the internet" (93\%), then meeting (91\%) or emailing (90\%) immediate colleagues. Directly contacting researchers or commissioning university researchers were ranked the lowest in importance. At the state level, only $18 \%$ of respondents claimed that using research evidence was a "low priority" in their unit, but a majority claimed there was insufficient time to read research (57\%) or build relationships with researchers (56\%). A smaller study by Ritter (2009) interviewed 31 policymakers on research use in drug policy and found the overall most common source of evidence was direct contact with an expert. Among the least common, as in Head et al's survey, was academic literature directly, which is likely related to reported frustrations around the contradictory and complex nature of academic literature (Ritter, 2009). Ritter suggests that, in light of the knowledge that multiple processes and pressures are involved with modern policymaking, "it seems important to maximise the chances that research evidence will at least be available and accessible" (Ritter, 2009: 71) for use by policymakers.

\section{Accessibility of evidence}

A significant barrier to effective research use outside of the academy in general is possibly the accessibility of academic literature. Head et al. (2014: 91) observe "collaboration and interaction across these sectors would require adjustments and efforts from both sides", in both incentives given to policymakers and to researchers. Ritter draws attention to a "dissonance between what is rewarded within the academic community and what is valued by policy makers", specifically difficulties in accessibility in publishing, the lack of policy-relevant summaries and language, and the sometimes discordant research questions of policy and academic research (Ritter, 2009). Within this is a major argument for the push for open access publication, leading to measureable impact increase (Archambault, 2010). However, while research exists on the impact of open access publication on internal academic discussion, little is known regarding open access publication's effect on the impact of research outside the academy. The literature so far on open access impacts within the research community shows a moderate positive effect but also amplifies competition, creating "losers as well as winners" (Mccabe et al., 2013). There is also evidence that authors in a variety of disciplines are adopting open access practices and being rewarded for doing so by traditional impact measures such as citations (Antelman, 2004). The most recent comprehensive studies on the proportions of open access publishing include a review conducted across 2004 to 2011 (Archambault et al., 2013) and one which samples from 2009 and 2011 (Gargouri et al., 2012) which both found a distinct difference in publishing practice across different research fields. Notably, prominent areas aligned with policy 
departments, such as social sciences, have a low open access proportion, where characteristically publishing open access has a larger impact advantage (Archambault et al., 2013). Publishing open access research in these fields may have a so far uninvestigated effect on research use in those policy domains.

\section{Gaps and implications}

Strikingly, across the literature for research use in policy there is a bias for detailed studies within health fields. While these field-specific, more thorough studies produce clear pictures of research use within a community, the few large-scale studies that have been conducted show differences in approaches between fields, so possibly not all of these conclusions apply to all policy domains. Similar detailed studies in different fields or across fields is clearly necessary. Overall, studies incorporating different fields, longitudinal data and questions probing how research is used at each stage of the decision-making process have been recommended (Amara, Ouimet and Landry, 2004; Head et al., 2014). Previous studies also rely on interview and survey data, which while giving a breadth of information regarding attitudes to research, can be subject to biases such as volunteer or recall bias (Basit, 2017). These methods also capture general thinking towards research use, and not the direct measurable use per policy or publication. Citation analysis does not capture this broad spectrum, but citations are are a common surrogate for impact (Antelman, 2004), and are an important part of the research machinery that should not be ignored. The public statement of what evidence was utilised where, measured as standard through citation and other growing bibliometric methods, is a necessary balance and a foil to the comprehensive data gathered through qualitative studies. At the citation level, we can also investigate the impact of open access publishing of academic evidence.

In the past, citation analysis in the policy sphere has been avoided likely due to lack of available data, but the start-up Altmetric recently released data on academic publications mentioned in "policy-related" documents, such as from the European Food Safety Authority, the United Kingdom Government, and the World Health Organization, which allowed for some of the first bibliometric studies of impact of research in policy. Two pilot studies were conducted, testing a sample population of journal articles for citation in the released policy-related documents (Bornmann, Haunschild and Marx, 2016; Haunschild and Bornmann, 2017). These studies found very low rates of citation, with only $0.5 \%$ overall, and only $2 \%$ of climate-change related articles being cited in policy-related documents. The authors hypothesise this could be because the coverage released by Altmetric was quite small, there may be a different citation culture outside of scientific research, and that perhaps there is only a small part of scientific literature that is relevant to policy (Haunschild and Bornmann, 2017). The reverse case to these studies - analysing a corpus of policy documents for the proportion of academic references - has, however, seemingly not been conducted. There seems to be growing interest in this sort of analysis, as shown in the work surrounding Altmetric's data release, and the increased coverage on Australian policy document database the Analysis \& Policy Observatory (2017).

\section{This study}

To investigate the variety and balance of knowledge sources cited by policymakers in Australia, as well as analyse the impacts of accessibility of these sources, we conducted a bibliometric study of citations of recent publications from a sample of Australian Federal Government departments. This assessed on a wide cross-sectional basis the proportion of internal and external knowledge specifically cited in publication. Government publications are reports, documents and other informative resources issued by Australian Commonwealth bodies, and are a main output of policy-aimed information for 
many departments. By assessing evidence cited, further inferences can be drawn regarding specific types of evidence used, regarding recency, location, and accessibility of research, as well as the differences across departments and policy domains. Data on how frequently policymakers read academic sources for policy, and others from interviews, grow more useful when we also know whether or not those same proportions end up in public sight as cited evidence in policy documents. Thus, this analysis of information cited by the Australian Federal Government allows for significant headway in understanding what specific knowledge sources are legitimately sought for policymaking, even at a late stage to support existing policy decisions. It also serves as a sample review of Australian Government publications in general, as well as accessibility of Government publications, and adds to the justification of a comprehensive database and index of policy documents.

\section{Methods}

\section{Study population}

Publications from policy-focused Australian Government departments were chosen to investigate policymaking, as actual party policy and bills rarely include citations, but published publications include documents which influence or are representative of policy processes. There were eleven departments classified as "large", consisting of over one thousand employees, and "policy-focused" in the 2011-2012 State of the Service report (Australian Public Service Commission, 2012), which claims to be the most up-to-date classification. After mapping these to the modern department domains and attempting to find online publications, eight departments were chosen for inclusion in the study: the Attorney-General's Department, the Department of Education and Training, the Department of the Environment, the Department of Foreign Affairs and Trade, the Department of Health, the Department of Infrastructure, the Department of Social Services and the Department of the Treasury. As Head et al., (2014) found no significant difference in practices between state and federal governments, only federal departments were investigated in this study. However, multiple studies (Amara, Ouimet and Landry, 2004; Ritter, 2009; Head et al., 2014) found a difference in organisational environments and field of research, so the spread of publications were collected across federal policy departments rather than a larger set from a single department. The ten most recent referenced publications available from each department were collected, with a cut-off date of 23 January 2017. A publication was said to include references if it had at least one full reference, excluding explanatory footnotes or simple links to further information. A larger sample was outside the scope of this project, exacerbated by the difficulty of finding older publications from department websites and the differences in publishing standards between departments.

\section{Information coding}

Multiple fields of information were recorded for the eighty total publications and the references from each. A coding system based on Miles and Huberman (1984) was used to categorise the publications and references, starting with a provisional list of categories from prior studies, then distilling final categories from the collected data. For each publication, we recorded the author, title and year and categorised a publication 'type', based on whether it was authored internally or externally, and whether it was developing or evaluating policy. 
For each reference from the publications, we manually collected the title and authors of the cited work for redundancy identification along with the year, location, the title of the book or journal it was published in, and categorised the citation under sixteen discrete source types: Australian business, Australian federal government, Australian government consultant, Australian organisation, Australian state government, Australian university, book, conference, foreign business, foreign government, foreign organisation, foreign university, intergovernmental, media article, peer-reviewed journal, and other. Journal articles and books or book sections were simply categorised. Government categories were complicated, as varying referencing styles across publications made authorship sometimes ambiguous. Foreign government reports and state government reports were labelled as such, then reports either with department names or author lists as authors were labelled 'government', with distinction from 'government consultant' reports where consulting firms or groups were listed as author. Sources such as 'business' or 'organisation', split into Australian and non-Australian ('foreign'), were best coded by the information within the reference such as a .com, .com.au, .org, or .org.au web domain or information available in the evidence cited, and otherwise left categorised wholly as 'other'. A category for intergovernmental reports was also included. Each source was only recorded once per publication, as most sources cited more than once in a single publication were for technical rather than substantive reasons, such as citing survey data. Sources cited in multiple publications were recorded multiple times. Responding to the assumptive characterisation of citations as purely positive acknowledgements of research use, we initially noted the text surrounding each citation. However, after investigating a subset of the publications, there was not sufficient information from the publication text to discern citation sentiment.

For peer-reviewed journal articles, we also recorded the availability of the source, following the common distinctions of green and gold access levels (Gargouri et al., 2012). Gold refers to an entirely open access journal, while green refers to articles that are either paid to be made open access, or later released online elsewhere. References that appeared to be from peer-reviewed journals, but for which we could track down no further information, were coded with 'unknown' access, and excluded from further analysis.

\section{Results}

The final study population consisted of 4649 references collected from 80 publications across eight government departments spanning from 2010 to 2017 , though all were uploaded or re-uploaded to departmental websites from 2015 to 2017 . The following section presents and briefly discusses the characteristics of the 80 publications, the 4649 references, and the 1836 referenced journal articles, before further research discussion and recommendations.

\section{Collected publications}

Table 1: Total references $(n=4649)$ from the collected ten most recent referenced Australian Government publications, by department and author type

\begin{tabular}{|l|l|l|l|}
\hline Department & $\begin{array}{l}\text { Number of } \\
\text { Government- } \\
\text { authored } \\
\text { publications }\end{array}$ & $\begin{array}{l}\text { Number of } \\
\text { consultant- } \\
\text { authored } \\
\text { publications }\end{array}$ & $\begin{array}{l}\text { Number of } \\
\text { references } \\
\text { from } \\
\text { publications }\end{array}$ \\
\hline Attorney General's Department & 7 & 3 & 508 \\
\hline
\end{tabular}




\begin{tabular}{|l|l|l|l|}
\hline Department of Education and Training & 5 & 5 & 306 \\
\hline Department of Foreign Affairs and Trade & 9 & 1 & 434 \\
\hline Department of Health & 8 & 2 & 1538 \\
\hline Department of Infrastructure & 10 & 0 & 519 \\
\hline Department of Social Services & 8 & 2 & 230 \\
\hline Department of the Treasury & 9 & 1 & 324 \\
\hline Department of the Environment and Energy & 5 & 5 & 790 \\
\hline Totals & $\mathbf{6 1}$ & $\mathbf{1 9}$ & $\mathbf{4 6 4 9}$ \\
\hline
\end{tabular}

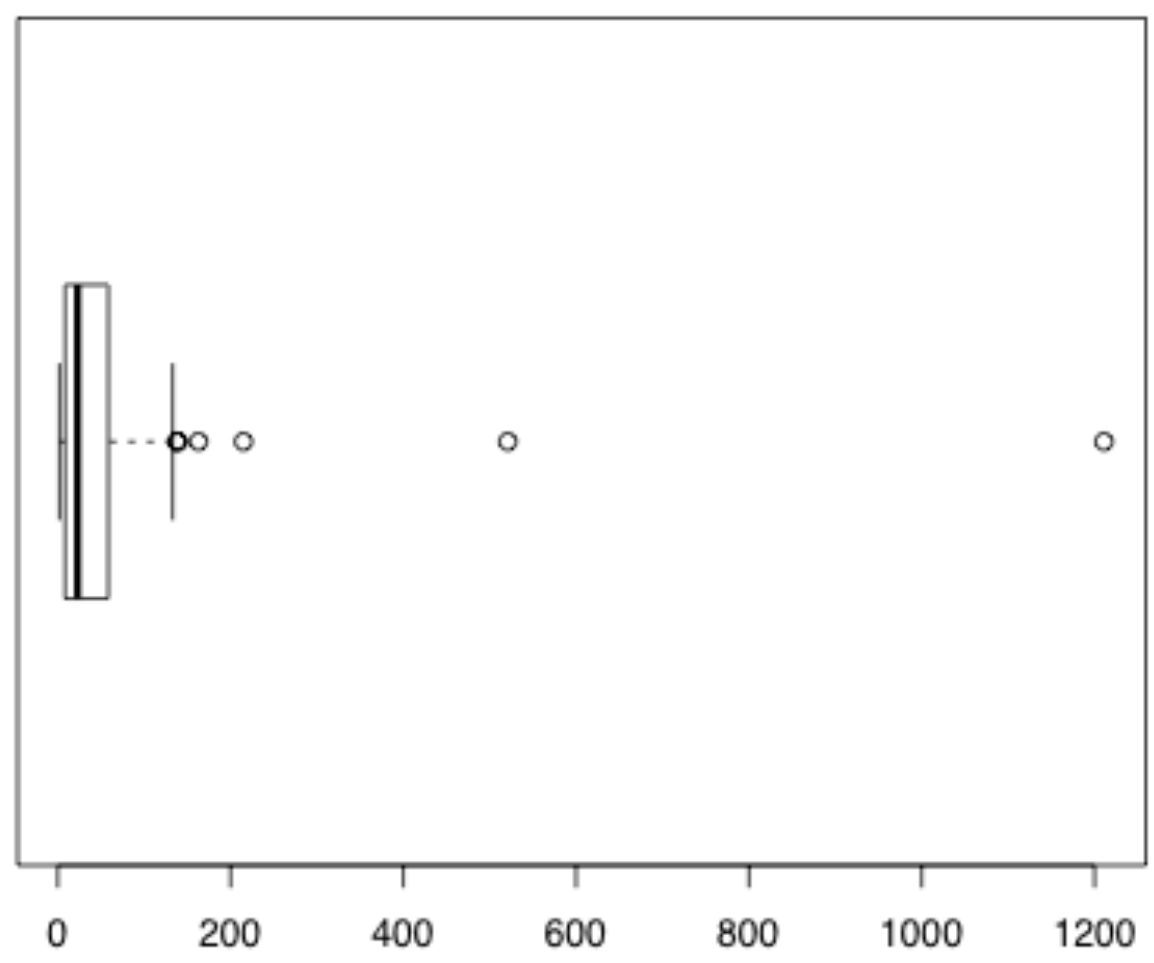

Figure 1 Total reference counts per publication (minimum: 2, median: 23, mean: 58, max: 1211, $n=4649$ )

As this is a relatively small, cross-sectional study, the collected publications were varied in both the publication author type and the number of references in each, as seen in Table 1. The median number of references for a publication was 23, with an average of 58, and a standard deviation of 147 (Figure 1). A similar spread was seen within each department. At the extreme, the Department of Health had one report with 1200 references, the effects of which are seen in more detail in the next section.

Publications were only collected from eight departments, out of a total of eleven large, policyfocused departments. Primarily, this was due to how easily accessible the publications were online, or in some cases the structure of the department; for example, the Department of Industry, Innovation and Science publishes publications under subservient agencies, not the Department itself. The collected publications are dated from 2010 at the oldest and 2017 at the most recent, and included research, development reports, evaluations, policy and guidelines by both government departments 
and consultants. The inclusion dates were taken from the date published to the department website, which sometimes did not match the publication date. The actual publication date was used for further analysis once it was included in the study.

\section{Collected references}

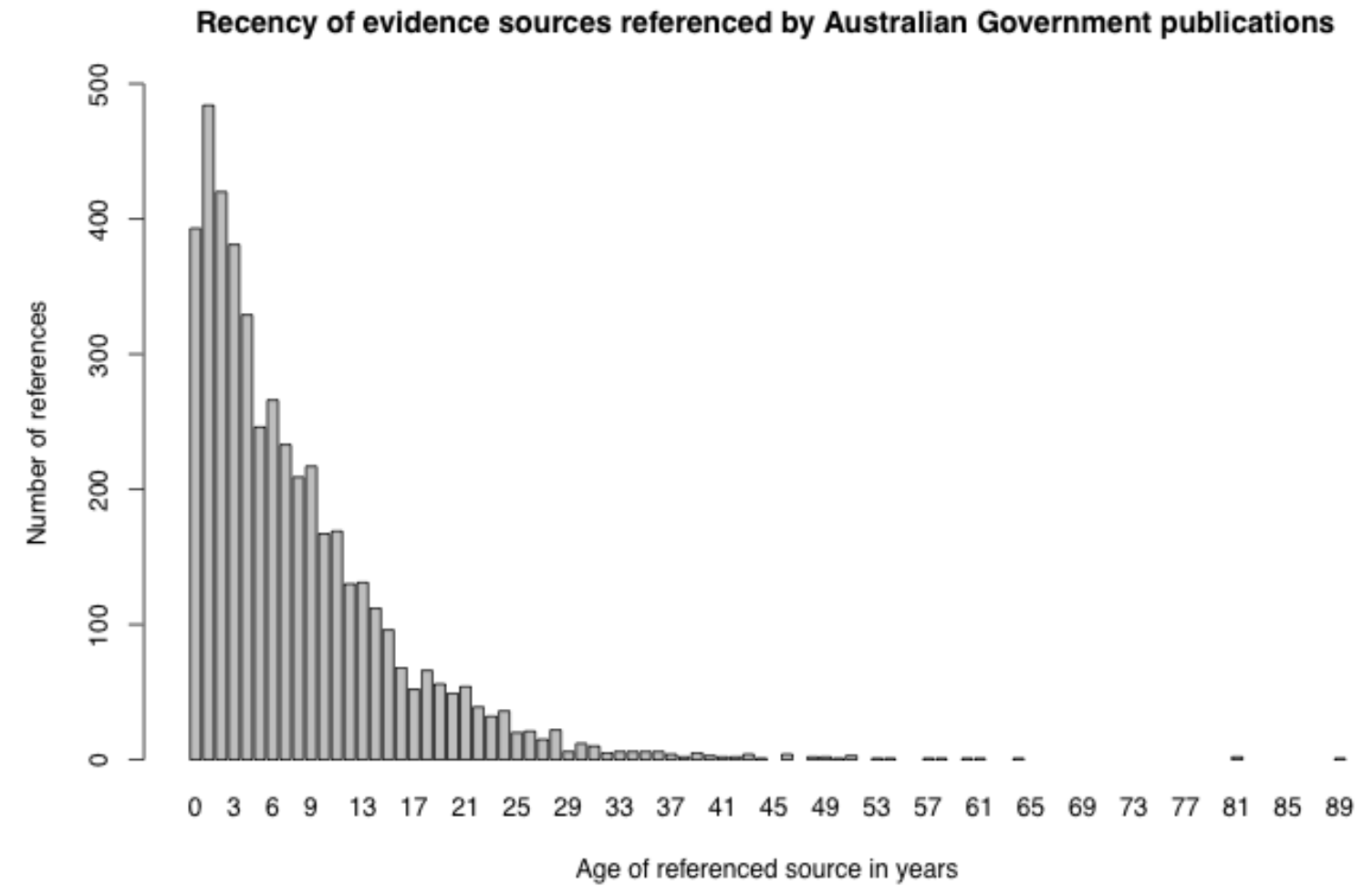

Figure 2 Total number of references $(n=4649)$ by recency of source in years, all departments

There were 4649 references collected in total, each with a recorded author, title, year, parent publication and categorised source type. To analyse the recency of evidence use in policy, we calculated the age of each reference from the year of its citing publication, and graphed all 4649 references per age in years (Figure 2). The recency of the information follows roughly an exponential decay curve. The curves for each individual department followed roughly the same shape, however, as there is a great difference in number of references across departments that we collected, comparing these directly was not viable. 


\section{Evidence source types referenced by Australian Government publications}

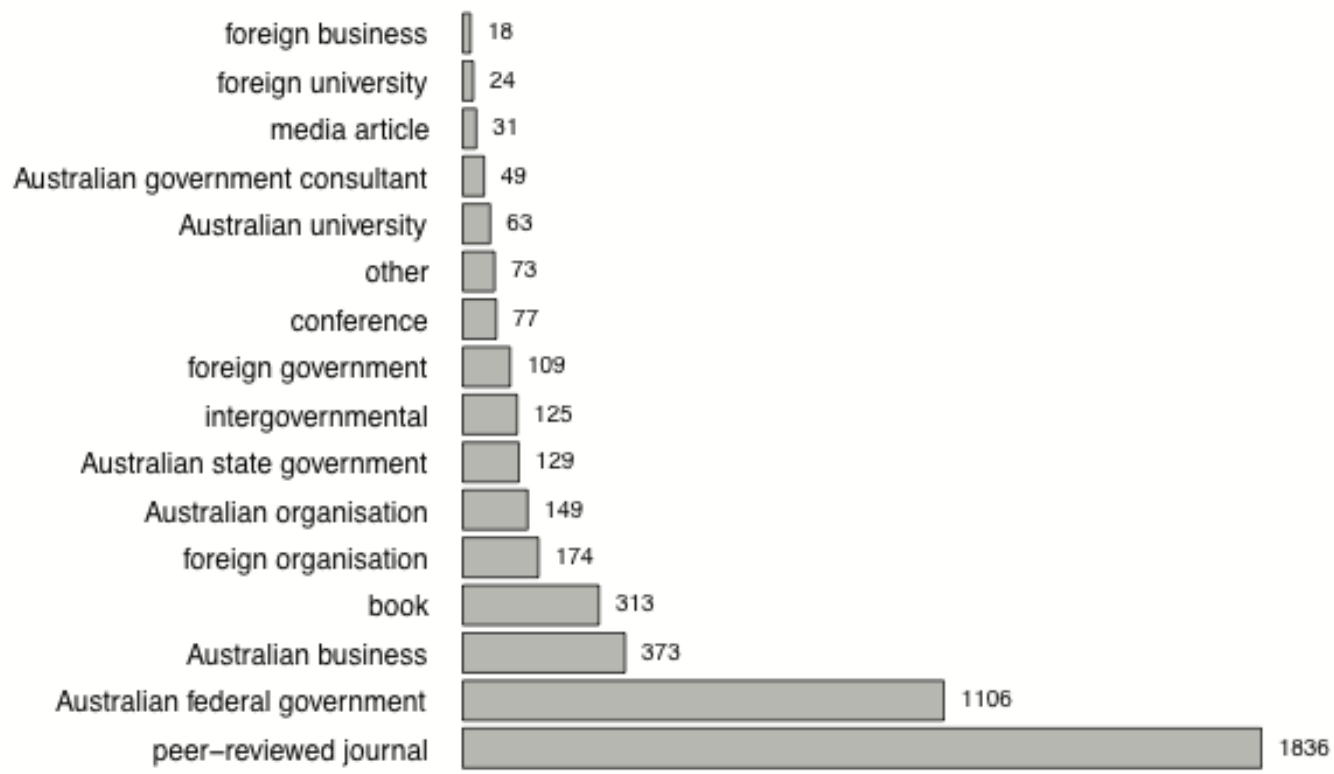

Number of references

Figure 3 Evidence source types cited by Australian Government publications, all eight departments, $n=4649$

\section{Evidence source types referenced by Australian Government publications, by department}

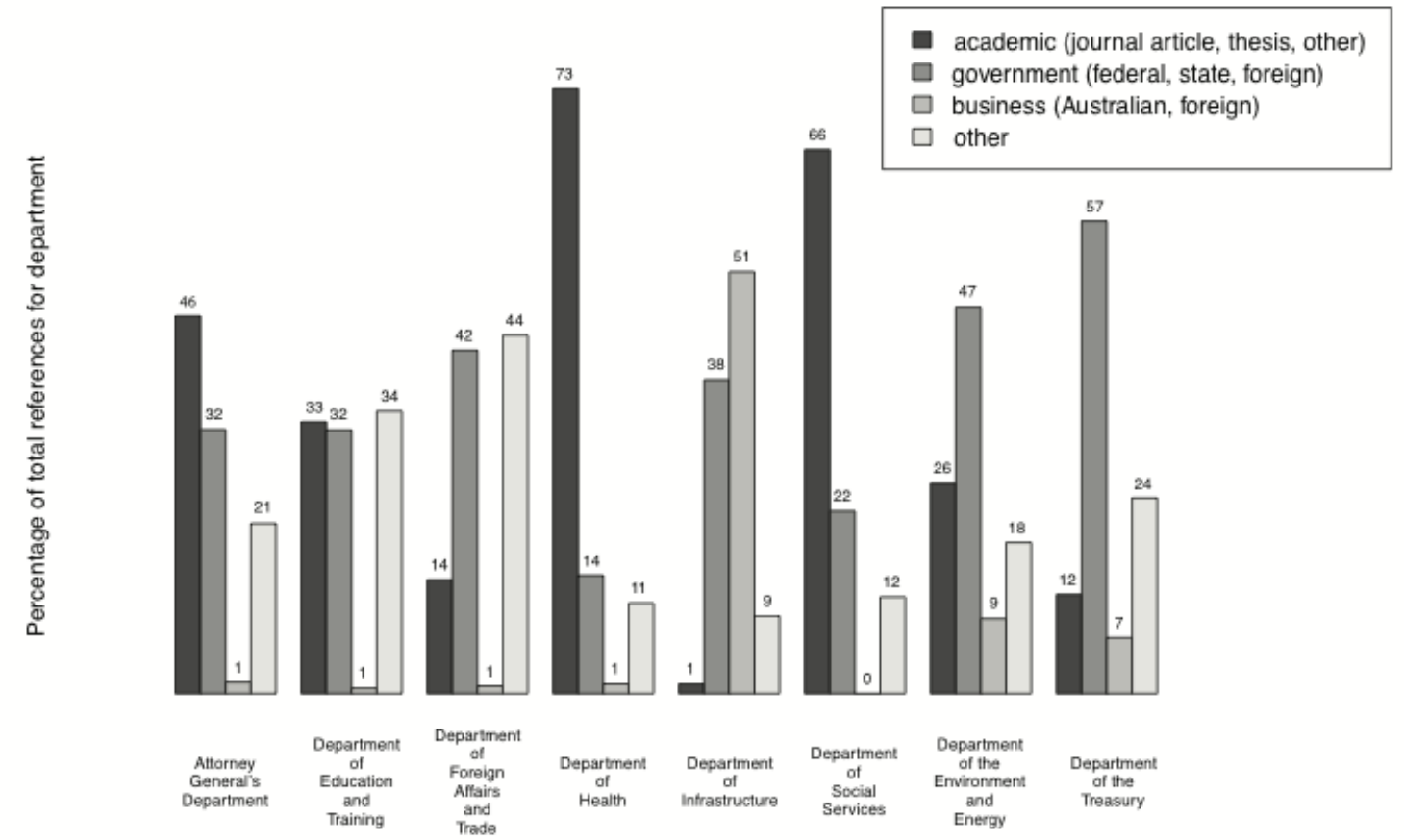

Figure 4 Source type of cited evidence by department, grouped by academic, government, and business categories, $n=4649$

Categorisation of the evidence types was determined without external labels, and thus was decided to describe the balance of sources in the data itself. Sixteen categories were selected for coding each reference's source type. They were chosen to best cover the primary categories of evidence sources 
used, though not all of the categories were distinct and differences in referencing styles from department to department and from publication to publication made categorisation difficult. Breaking down the 'other' category further was not illuminating, as it included mostly items with no discernible source, or missing information. For figure 4 above, the sixteen categories in figure 3 were grouped to broader versions of the three highest overall sources of evidence: academic, government and business. Academic sources included journal articles and other published university research; government sources included federal, state, and foreign government reports as well as intergovernmental reports; business sources included both Australian and foreign business information; the 'other' category included all other references.

The most common source type overall was peer-reviewed journal articles, followed by Australian federal government publications, then Australian business information. Each department varied slightly in their major source types, all of which seem to suit the separate policy domains. The Department of Infrastructure, reporting primarily on transport and infrastructure projects, has a large proportion of references to business information. The Department of Foreign Affairs and Trade has a large proportion of information from foreign governments and intergovernmental reports. The Attorney General's Department, the Department of Health, and the Department of Social Services all lean most on academic sources. The single publication from the Department of Health had a profile itself of a high $79 \%$ peer-reviewed journal articles, with the next highest $8 \%$ books and $4 \%$ government sources, which clearly had an effect on the profile of the Department of Health sources, and the overall sources. However, general conclusions can be drawn from, if not the specific absolute counts or proportions, but the rankings of evidence sources. Excluding the report does not alter the top three sources, though they alter from journal articles (39\%), Australian federal government reports (24\%), then Australian business information (8\%), to Australian federal government reports (30\%), journal articles $(25 \%)$ and business information (10\%). Similarly, excluding the report also specifically alters the Department of Health top sources from journal articles (72\%), government reports $(11 \%)$ and books $(6 \%)$ to journal articles $(44 \%)$, government reports $(36 \%)$ and reports from Australian organisations (6\%). These skews must be kept in mind while drawing conclusions from this data, however, as this single Department of Health report is the largest outlier, the general rankings of evidence sources are still useful, especially in departments with less variance in the number of references.

\section{Journal article references}

The proportion of peer-reviewed journal articles amongst source types in government publications was relatively high, though it differed substantially across departments. Splitting the references by department resulted in groups ranging from two references to 1102 references, with the bulk of the largest group coming from a single publication, but not all of the groups consisting of research from similar fields. Therefore, analysis was conducted with the entire population of referenced journal articles in groups based on research subject. This was also in order to compare article availability with a separate review of open access publishing (Archambault et al., 2013) seen in Table 2.

To construct this comparison, we labelled each referenced article by journal subject as listed by the SCOPUS database (Elsevier, 2017), then grouped the articles by subjects matching those listed by Archambault et al. we calculated a predicted open access publication proportion for 2012 to 2015 by applying Archambault et al's calculated static growth rate from 2004-2007 to 2008-2011 to their open access proportion for 2008-2011. We then compared this number to the proportion of open access publications in the collected references. 
Table 2 Proportion of open access articles cited in government publications compared to predictions of overall open access publishing proportions, based on data from Archambault et al. (2013)

\begin{tabular}{r|lll} 
Journal subject & $\begin{array}{l}\text { Total } \\
\text { articles }\end{array}$ & $\begin{array}{l}\text { Open access \% of } \\
\text { referenced articles } \\
\text { in a sample of } \\
\text { Australian } \\
\text { Government } \\
\text { publications }\end{array}$ & $\begin{array}{l}\text { Predicted open } \\
\text { access \% from } \\
\text { Archambault, et al } \\
\text { (2013) }\end{array}$ \\
\hline Clinical Medicine & 820 & 59 & 55 \\
Psychology \& Clinical Sciences & 303 & 56 & 50 \\
Social Sciences & 299 & 47 & 36 \\
Agriculture, Fisheries \& Forestry & 121 & 52 & 50 \\
Economics \& Business & 76 & 67 & 46 \\
Earth \& Environmental Sciences & 64 & 56 & 43 \\
Public Health \& Health Services & 55 & 57 & 58 \\
Biomedical Research & 35 & 69 & 63 \\
Information \& Communication Technologies & 21 & 62 & 46 \\
General Arts, Humanities \& Social Sciences & 15 & 60 & 31 \\
General Science \& Technology & 9 & 78 & 62 \\
Biology & 7 & 71 & 40 \\
Chemistry & 5 & 40 & 46
\end{tabular}

\section{Discussion}

The set of collected references was analysed both as a whole, and separate departmental groups, and compared to reference practice in the core research community and previous knowledge of reference practice in policymaking. It is difficult to know if the collected publications are representative of the usual output of government publications across a similar time period, or if these publications are directly relevant to policymaking or not. Most publications seemed at least intended to assist with policy, such as national framework evaluations or policy development research. As the publications are taken from eight separate departments from a number of years, the sample is at least indicative enough to note estimates of current proportions, though not definite trends across the departments or years.

Organisational differences may explain at least the variance in reference counts between departments. As previous studies (Rich and Oh, 2000; Head et al., 2014) have found, a difference in 
organisational culture can lead to a different approach to research use. In the narrow realm of citations, a difference in not only approach to research but approach to referencing practice could be affecting the number of references per publication across departments or even across consulting authors. In addition, lengths of publications differing non-randomly across departments may have an effect, as some departments may consistently output long publications, and logically these would have more information to reference.

The age of each source was calculated to observe the overall recency of cited evidence. The general decay curve of age of references seen in figure 2 is well-documented in the core research community, differing in steepness across research fields (Macrae, 1969). It is understandable that government research follows this trend, especially so as policy should take into account the most recent evidence; it is difficult to tell with this data how the decay rate compares directly to other research communities or fields. The off-trend decrease in references for evidence younger than 2 years is likely due to a lack of research available as well as the speed of publishing both of government publications and the cited evidence, as on average each publication would only have research available from half of the current year, and many published articles have embargoes of 12 months.

Regarding sources of cited evidence, while internally the balance of sources may suit each department's focus, the rankings of evidence source types do not align with previous studies into evidence use in policy in Australia. Head et al. (2014) and Ritter (2009) both found that the most common source of evidence was direct correspondence with a colleague or expert, while the categories represented highly here, such as government reports or academic literature, are ranked after five other sources. There are a few clear explanations for this difference, though we cannot be certain of their validity or pervasiveness here. Most importantly, the categories of previous studies do not map precisely to actual evidence sources, preferring to focus questions on the actions of policymakers as they research. For example, while citation analysis can separate using evidence from a government website or a business's website, past surveys have categorised both of these under "searching the internet" (Head et al., 2014). Similar groupings exist for the highest-ranked categories, mostly talking directly to an expert. In addition, asking about evidence use in the research process is fundamentally different to asking about evidence in the citation process. This manifests in a few ways. Firstly, policymakers may claim during interviews that a certain source is their first step, or most important to the research process in general, but it may not lead to any instrumental information that they later cite. Secondly, actions such as speaking directly to an expert may not be an evidence source itself, but may point policymakers to other evidence sources, which are then cited. In a related issue, even when the expert may be a direct source of evidence, policymakers may not know to cite that correspondence, or feel comfortable doing so. Certainly, despite policymakers across the country reportedly ranking direct correspondence very highly in their research, only one clear reference to personal correspondence was found in any of the 80 publications. If policymakers view citations as a more symbolic use of research, an almost reflexive support of existing ideas or statements, rather than an acknowledgement of original sources, this could result in the citations of a policymaker differing quite broadly from what they recount in interviews about the research process. This gap is certainly present in academic research (MacRoberts and MacRoberts, 1996), not just a symptom of policy research, though it may be stronger from those without an academic background. This could be related to prior findings regarding the association between individual policymaker characteristics and their use of research (Lester, 1993; Head et al., 2014), as policymakers with an academic background may have different understandings of the purpose of citation. Recommendations on the best communication channels to use as a researcher looking to affect policy are based on our understanding of the highest-ranked sources policymakers seek. If there are discrepancies in our understanding, clear best practice cannot be stated. This gap in results is an important oversight in studies of research use, particularly in policy, and could be further investigated with directly linked interviews and citation analyses. 
The references to peer-reviewed journal articles were separately analysed to investigate the effect of open access publishing on the likelihood of being cited in policy documents. The use of evidence from peer-reviewed journal articles differed by department, likely due to both differences in organisational or contextual perspectives on research, and the different policy domains, as discussed above. The proportions of open access articles, seen in table 2 split up by research domain, clearly match up to the general trends across domains in the broader literature, but at an average of 11 percentage points higher when compared to the predictions calculated according to the measured growth index. This is not particularly rigorous comparison; in brief, it is a small body of data, the articles were categorised not per article but by SCOPUS subject per journal, the comparison data is older and not specifically Australian focused, and the predictions are based on a static growth rate. However, the review of 2004 to 2011 captures approximately $48 \%$ of the referenced articles in this study, with $83 \%$ published before 2011 , meaning a minority of articles likely would have been published under a higher growth rate environment. It is also important to consider that the evaluation of article accessibility was conducted for some articles many years after publication, while the comparison data is from the year of publication. As open access per-article increases slightly over time (Archambault et al., 2013) likely due to embargoes and delayed self-archiving, therefore the data might be slightly above the proportion at-publication. With all this considered, there is still a trend, most visible in the larger cohorts of clinical medicine, social sciences, economics and business, earth and environmental sciences, and general arts, humanities and social sciences, that the references selected as sources of evidence for government publications are more likely to be open access. Whether these two factors are correlated or indeed causally related at all is practically impossible to extract from this data, due to the reasons mentioned above and, importantly, a lack of a direct comparable group of publications that are open access but were not referenced. Regardless, impact studies from within the academic community point to open access publications having higher citations (Mccabe et al., 2013), and interviews with policymakers point to a focus on ease and availability of evidence (Ritter, 2009), something open access publications increases. This effect is possibly stronger within policy research than academic research, as policymakers may have decreased access to journal subscriptions. Determining the exact nature and extent of this effect would require a larger dataset, including an up-to-date comparison data on open access proportions, particularly in Australia and in policy-focused domains.

While a large number of references were collected in total, the number of references per department, and number of collected publications, was relatively small, meaning some comparative trends were difficult to determine with confidence. It would be worthwhile to investigate these differences with a larger dataset, comparing references per publication and per word count, or some similar measure, across departments and authors. Closer categorisation of publications such as commissioned author and purpose, which may involve contacting the department for information, could be worthwhile to compare more directly. A longitudinal dataset would allow for more meaningful tracking of data trends across years. Adding publications from the departments not included in this study due to accessibility issues with government publishing would increase the dataset size and allow for further interdepartmental comparison. With regards to method, combining citation analysis with specific interviews or surveys linked to each publication could build an understanding of differences in referencing practice.

\section{Conclusion}

This bibliometric study aimed to gain insight into the research and reference practices of Australian policymakers, and investigate how this approach compares to previous interview and survey studies. 
By analysing a small set of references from government publications, we have shown that despite the criticisms of citation analysis as an impact measure, at least in the field of research utilisation we cannot solely rely on interview or survey data, as cited evidence use differs from reported evidence use. This is likely true to some extent of all researchers, but citation analysis of policy documents certainly lags behind those of academic communities. Additionally, it is clear that the balance of evidence sources in policy differs from other research communities, and is worth investigating further, which would involve increased accessibility of government publications and references. Specifically, analysing the references of a larger set of publications longitudinally would grant the ability to mark trends and more confidently compare contexts across departments and policy domains, and over time.

\section{References}

Amara, N., Ouimet, M. and Landry, R. (2004). New Evidence on Instrumental, Conceptual, and Symbolic Utilization. Science Communication, 26(1), pp. 75-106. doi: $10.1177 / 1075547004267491$.

Antelman, K. (2004). Do Open-Access Articles Have a Greater Research Impact? College \& Research Libraries, 65(5), pp. 372-382.

Archambault, É. (2010). The Tipping Point - Open Access Comes of Age.

Archambault, E., Amyot, D., Deschamps, P., Nicol, A., Rebout, L. and Roberge, G. (2013). Proportion of Open Access Peer-Reviewed Papers at the European and World Levels-20042011.

Analysis \& Policy Observatory. (2017). Available at: http://apo.org.au/

Australian Public Service Commission. (2012). Appendix 2 - APS agencies. Available at: $\mathrm{http} / / / \mathrm{www} . a p s c . g o v . a u / a b o u t-t h e-a p s c / p a r l i a m e n t a r y /$ state-of-the-service/2011-12sosr/appendix-2-aps-agencies.

Basit, T. (2017). Manual or electronic? The role of coding in qualitative data analysis. Educational Research, 45(2), pp. 143-154. doi: 10.1080/0013188032000133548.

Bollen, J., Sompel, H. Van De, Smith, J. A. and Luce, R. (2005). Toward alternative metrics of journal impact: A comparison of download and citation data. Information Processing \& Management, 41, pp. 1419-1440. doi: 10.1016/j.ipm.2005.03.024.

Bornmann, L., Haunschild, R. and Marx, W. (2016). Policy documents as sources for measuring societal impact. Scientometrics, 109(3), pp. 1477-1495. doi: 10.1007/s11192-016-2115-y.

Clarivate Analytics. (2017). Journal List, Science Citation Index. Available at: http://ipscience.thomsonreuters.com/cgi-bin/jrnlst/jlresults.cgi? $\mathrm{PC}=\mathrm{K}$.

Curtin University. (2017). Measure research impact and quality. Available at: http://libguides.library.curtin.edu.au/research-impact

Deakin University. (2016). Demonstrate your research impact. Available at: http://www.deakin.edu.au/library/research/demonstrate-your-research-impact.

Department of Education Training \& Youth Affairs. (2000). The Impact of Educational Research.

Department of Industry Innovation and Science. (2017). Measuring impact and engagement of university research. Available at: http://www.innovation.gov.au/page/measuring-impact-andengagement-university-research.

Dolenc, J., Hünenberger, P. and Renn, O. (2016). Metrics in Research: For better or worse?. Infozine $S-1,(1)$.

Elsevier. (2017). Content. Available at: https://www.elsevier.com/solutions/scopus/content.

Gargouri, Y., Larivière, V., Gingras, Y. and Harnad, S. (2012). Green and Gold Open Access Percentages and Growth, by Discipline. 17th International Conference on Science and Technology Indicators. Montreal.

Haunschild, R. and Bornmann, L. (2017). How many scientific papers are mentioned in policy- related documents? An empirical investigation using Web of Science and Altmetric data. Scientometrics, 110(3), pp. 1209-1216. doi: 10.1007/s11192-016-2237-2.

Head, B., Ferguson, M., Cherney, A. and Boreham, P. (2014). Are policy-makers interested in social research? Exploring the sources and uses of valued information among public servants in 
Australia. Policy and Society, 33(2), pp. 89-101. doi: 10.1016/j.polsoc.2014.04.004.

King's College London and Digital Science. (2015). The nature, scale and beneficiaries of research impact: An initial analysis of Research Excellence Framework (REF) 2014 impact case studies.

Lester, J. P. (1993). The Utilization of Policy Analysis by State Agency Officials. Knowledge: Creation, Diffusion, Utilization, 14(3), pp. 267-290.

Levitt, J. M. and Thelwall, M. (2008). Is Multidisciplinary Research More Highly Cited? A Macrolevel Study. Journal of the American Society for Information Science and Technology, 59(12), pp. 1973-1984. doi: 10.1002/asi.

Macrae, D. (1969). Growth and Decay Curves in Scientific Citations. American Sociological Review, 34(5), pp. 631-635.

MacRoberts, M. and MacRoberts, B. (1996). Problems of citation analysis. Scientometrics, 36(3), pp. 435-444. doi: 10.1007/BF02129604.

Mccabe, M. J., Snyder, C. M., Kirk, E., Moxnes, A., Müller-langer, F., Pavcnik, N. and Quigley, J. (2013). Identifying the Effect of Open Access on Citations Using a Panel of Science Journals. 17.

Miles, M. B. and Huberman, A. M. (1984). Qualitative data analysis: A sourcebook for new methods. Newbury Park: Sage.

Norris, M., Oppenheim, C. and Rowland, F. (2008). The Citation Advantage of Open-Access Articles. Journal of the American Society for Information Science and Technology, 59(12), pp. 19631972. doi: 10.1002/asi.

Ouimet, M. and Ziam, S. (2009). The absorption of research knowledge by public civil servants. Evidence \& Policy, 5(4), pp. 331-350.

Rich, R. F. and Oh, C. H. (2000). Rationality and Use of Information in Policy Decisions. Science Communication, 22(2), pp. 173-211.

Ritter, A. (2009). How do drug policy makers access research evidence? International Journal of Drug Policy, 20, pp. 70-75. doi: 10.1016/j.drugpo.2007.11.017.

Saha, S. (2003). Impact factor : a valid measure of journal quality? Journal of the Medical Library Association, 91(1), pp. 42-46.

Universities Australia. (2016). Universities Australia Submission to the Engagement and Impact Assessment Consultation Paper.

Wallin, J. A. (2005). Bibliometric methods: Pitfalls and possibilities. Basic and Clinical Pharmacology and Toxicology, 97(5), pp. 261-275. doi: 10.1111/j.1742-7843.2005.pto_139.x.

Weiss, C. H. (1980). Knowledge Creep and Decision Accretion. Knowledge, I(3), pp. 381-404. 